\title{
Multi-region relaxed magnetohydrodynamic stability of a current sheet
}

\author{
J. Loizu ${ }^{1}$ and S. R. Hudson ${ }^{2}$ \\ 1 École Polytechnique Fédérale de Lausanne, Swiss Plasma Center, CH-1015 Lausanne, Switzerland \\ 2 Princeton Plasma Physics Laboratory, PO Box 451, Princeton NJ 08543, USA
}

(Dated: March 1, 2019)

\begin{abstract}
It is shown that the resistive MHD stability of a slab force-free current sheet can be calculated using the variational principle of multi-region relaxed magnetohydrodynamics (MRxMHD) and that the corresponding stability boundary is in exact agreement with linear tearing mode theory.
\end{abstract}

Over a decade ago, the theory of multi-region relaxed magnetohydrodynamics (MRxMHD) was proposed by Hole, Hudson and Dewar $[1,2]$ in an attempt to connect Taylor's relaxation theory [3] (which assumes global relaxation of the plasma and cannot describe equilibria with pressure gradients) and ideal MHD [4] (which imposes a continuous topological constraint on the magnetic field and cannot describe the formation of islands and chaos). In all three theories, macroscopic equilibria are obtained by extremizing the classical MHD energy functional [5],

$$
W=\int_{V_{p}}\left(\frac{p}{\gamma-1}+\frac{B^{2}}{2 \mu_{0}}\right) d V
$$

but with very different constraints. Here $V_{p}$ is the plasma volume and $\gamma$ is the adiabatic index. In MRxMHD, the plasma is partitioned into $N$ nested volumes separated by $N-1$ ideal interfaces that are assumed to remain magnetic surfaces during the minimization of the energy. In each volume, the plasma undergoes Taylor relaxation, namely the magnetic helicity

$$
K=\int_{V} \mathbf{A} \cdot \mathbf{B} d V
$$

is conserved within the volume along with the toroidal and poloidal magnetic fluxes. While varying the plasma potential energy, $W$, the ideal interfaces are allowed to undergo geometrical deformations and the resulting MRxMHD equilibrium states satisfy

$$
\begin{aligned}
\nabla \times \mathbf{B} & =\mu_{l} \mathbf{B} \\
{\left[\left[p+\frac{B^{2}}{2 \mu_{0}}\right]\right]_{l} } & =0
\end{aligned}
$$

where $l=1, \ldots N$ labels the volumes, $\mu_{l}$ is a constant characterizing the Taylor states, and $[[\cdot]]_{l}$ is the jump across the interface separating volumes $l$ and $l+1$. Equation (3) implies that the plasma pressure is constant in each volume. Equation (4) represents the local equivalent of the force-balance condition $\mathbf{j} \times \mathbf{B}=\nabla p$. In fact, it has been shown mathematically that MRxMHD retrieves exactly ideal MHD in the limit $N \rightarrow \infty$ [6]. And the case $N=1$ obviously retrieves Taylor's theory. MRxMHD allows to calculate three-dimensional, finite pressure, macroscopic equilibria in toroidal configurations, which generally exhibit regions of islands and magnetic field-line chaos [7]. The class of equilibria that MRxMHD can describe, which is restricted to steppedpressure profiles, has a solid mathematical foundation in that solutions are guaranteed to exist and with integrable plasma currents free of unphysical singularities $[8,9]$. We remark that, as of now, there are only a few other alternative classes of 3D MHD equilibria that are mathematically well posed [10-12].

The fact that MRxMHD is based on a variational principle suggests that while equilibrium states are found for which $\delta W=0$, their stability can also be evaluated by studying the sign of $\delta W$ for a finite perturbation around them. In the ideal limit, $N \rightarrow \infty$, we expect that an MRxMHD stability analysis will exactly retrieve the results of ideal MHD stability. Some numerical work has already confirmed this for a perturbed screw pinch equilibrium [13]. However, for finite $N$, it is expected that MRxMHD should also provide some information on potential instabilities that develop through spontaneous magnetic reconnection. In particular, tearing mode linear stability - and perhaps nonlinear saturation - may be described by MRxMHD.

In this Letter, we investigate the question of how MRxMHD stability relates to resistive MHD stability. We show that the tearing mode stability boundary for a slab current sheet is exactly retrieved by MRxMHD stability analysis, hence illustrating the potentially unifying approach of MRxMHD for the calculation of equilibrium and stability of partially relaxed plasmas.

We start by considering a force-free plasma current slab described by the following equilibrium profiles. A current density

$$
j_{z}(x)= \begin{cases}j_{z 0} & \text { if }-a<x<a \\ 0 & \text { if }|x|>a\end{cases}
$$

in the "toroidal direction", $z$, represents a current sheet of width $\delta=2 a$ in the "radial direction", $x$. The magnetic field corresponding to this current is, by virtue of Ampère's law, in the "poloidal direction", $y$,

$$
B_{y}(x)= \begin{cases}B_{y 0}^{\prime} x & \text { if }-a<x<a \\ -B_{y 0}^{\prime} a & \text { if } x<a \\ B_{y 0}^{\prime} a & \text { if } x>a\end{cases}
$$

where $B_{y 0}^{\prime}=\mu_{0} j_{z 0}$. Equations (5) and (6) imply that there is a $\mathbf{j} \times \mathbf{B}$ force in the radial direction, which must 
be balanced by the presence of a guide field $B_{z}(x)$ and the corresponding current $j_{y}(x)=-\partial_{x} B_{z}$. The equilibrium condition is the continuity of total pressure, namely $B_{z}^{2}+$ $B_{y}^{2}=$ const in this case. For a strong guide field, $B_{z} \gg$ $B_{y}$, the radial dependence of $B_{z}$ becomes very weak and the equilibrium in each of the three regions described by Eq. (6) approaches a Taylor state.

While this equilibrium is ideally stable [14], it is unstable to a tearing mode [15], and the magnetic energy can be lowered if a certain amount of magnetic reconnection is allowed at the resonant surface $x=0$. In fact, since $B_{y}(0)=0$, any perturbation with wavenumber $\mathbf{k}=\left(0, k_{y}, 0\right)$ will satisfy the resonance condition $\mathbf{k} \cdot \mathbf{B}=0$ at $x=0$. The instability threshold for the tearing mode is independent of resistivity and can be calculated from the linearized ideal MHD equations outside the resistive boundary layer. Generally, it is given by the sign of

$$
\Delta^{\prime}=\frac{1}{B_{x}}\left(\left.\frac{\partial B_{x}}{\partial x}\right|_{x=0^{+}}-\left.\frac{\partial B_{x}}{\partial x}\right|_{x=0^{-}}\right)
$$

with an instability occurring for $\Delta^{\prime}>0$. Here $B_{x}$ is the perturbed radial field for a given perturbation with wavenumber $\mathbf{k}$, and so $\Delta^{\prime}$ is generally a function of $\mathbf{k}$. For the current sheet equilibrium under consideration, and in the limit of a strong guide field, $\Delta^{\prime}$ can be obtained analytically [15] and is given by

$$
\Delta^{\prime} \delta=\frac{2 k \delta\left(e^{-k \delta}-k \delta+1\right)}{e^{-k \delta}+k \delta-1}
$$

where $k=k_{y}$ is the poloidal wavenumber of the perturbation. The conclusion is that this current sheet is always unstable to a tearing mode, $\Delta^{\prime}>0$, for sufficiently small $k$ or sufficiently small width $\delta$, as shown in Fig. 1. More precisely $\Delta^{\prime}>0$ for $k \delta \lesssim 1.28$. However, if we consider that the system is periodic in the poloidal direction, $y$, then the shortest possible wavenumber is $k_{\min }=2 \pi / L$ where $L$ is the length of the current sheet in the $y$ direction. The condition for instability thus becomes a condition for the current sheet aspect ratio,

$$
\frac{\delta}{L} \lesssim 0.2
$$

Tearing modes with larger wavenumbers, $k=m k_{\text {min }}$ with $m \in \mathbb{N}^{*}$, also become unstable for even thinner current sheets, namely for $\delta / L \lesssim 0.2 / \mathrm{m}$. This is illustrated in Fig. 2 where the stability threshold is shown for the $m=1$ and $m=2$ modes.

We now describe this current sheet equilibrium and its stability from the perspective of MRxMHD. In the limit of a strong guide field, the current density is piecewise constant, see Eq. (5). Thus we consider a threevolume MRxMHD equilibrium, Eqs. (3)-(4), with $\mu_{1}=$ $\mu_{3}=0$ for the outer volumes and $\mu_{2}>0$ determining the amplitude of the current density in the plasma. We also impose zero pressure, $p=0$, and assume that the equilibrium field as well as the geometry of the interfaces

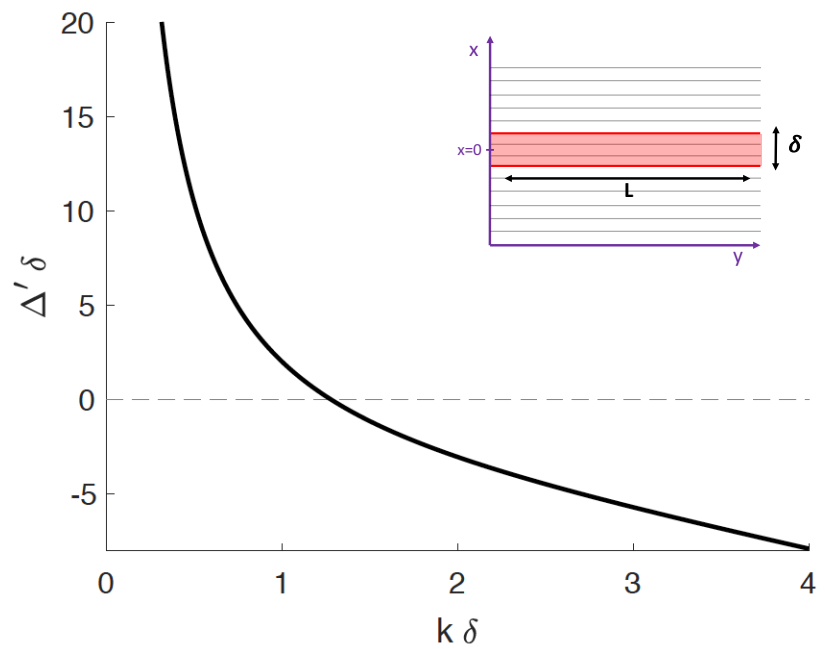

FIG. 1: Normalized threshold parameter for the tearing mode instability, $\Delta^{\prime} \delta$, as a function of the normalized perturbation wavenumber, $k \delta$, as obtained from Eq. (8). Inset: schematic view of the current sheet (shaded red) and the associated Poincaré plot of the field lines at constant $z$.

do not have any dependence on $y$ or $z$. In this case, the solution to Eq. (3) is analytical [10] and given by

$$
\begin{aligned}
& B_{y}(\bar{x})=\frac{\mu_{l}}{2 L}\left(\Psi_{t, l} \frac{\sin \left(\mu_{l} \bar{x}\right)}{\sin \left(\bar{\mu}_{l}\right)}+\Psi_{p, l} \frac{\cos \left(\mu_{l} \bar{x}\right)}{\sin \left(\bar{\mu}_{l}\right)}\right) \\
& B_{z}(\bar{x})=\frac{\mu_{l}}{2 L}\left(\Psi_{t, l} \frac{\cos \left(\mu_{l} \bar{x}\right)}{\sin \left(\bar{\mu}_{l}\right)}-\Psi_{p, l} \frac{\sin \left(\mu_{l} \bar{x}\right)}{\sin \left(\bar{\mu}_{l}\right)}\right)
\end{aligned}
$$

where $\bar{x}=x-\langle x\rangle_{l}$ and $\bar{\mu}_{l}=\mu_{l} \tilde{x}_{l}$. Here $\langle x\rangle_{l}=\left(x_{l}+\right.$ $\left.x_{l-1}\right) / 2$ and $\tilde{x}_{l}=\left(x_{l}-x_{l-1}\right) / 2$ are determined by the position of the ideal interfaces, $x_{l-1}$ and $x_{l}$, defining the boundaries of the relaxation volume $l$. For given values of the geometry, $x_{l-1}$ and $x_{l}$, the solution in volume $l$ is uniquely determined by three parameters, namely $\mu_{l}$ and the enclosed toroidal and poloidal magnetic fluxes, $\Psi_{t, l}$ and $\Psi_{p, l}$. The vacuum limit is well defined since $\sin (\mu) / \mu \rightarrow 1$ for $\mu \rightarrow 0$. The outermost interfaces, $x_{0}$ and $x_{3}$, are assumed to be fixed in order to prevent any forced reconnection [16]. The location of the internal interfaces, $x_{1}$ and $x_{2}$, is determined by the force-balance condition, Eq. (4), which can be written as

$$
f_{l}=f_{l}^{+}+f_{l}^{-}=0
$$

for $l=1,2$, and where

$$
\begin{aligned}
f_{l}^{+} & =\frac{1}{8 L^{2}} \frac{\mu_{l+1}^{2}}{\sin ^{2}\left(\bar{\mu}_{l+1}\right)}\left(\Psi_{t, l+1}^{2}+\Psi_{p, l+1}^{2}\right) \\
f_{l}^{-} & =-\frac{1}{8 L^{2}} \frac{\mu_{l}^{2}}{\sin ^{2}\left(\bar{\mu}_{l}\right)}\left(\Psi_{t, l}^{2}+\Psi_{p, l}^{2}\right)
\end{aligned}
$$

are the forces acting on each side of each interface. The equilibrium solution can be obtained by solving Eq. (12) provided that $\left\{\mu_{l}, \Psi_{t, l}, \Psi_{p, l}\right\}$ are given in each volume. 
We choose $\mu_{1}=\mu_{3}=0, \mu_{2}=0.1, \Psi_{t, 1}=\Psi_{t, 3}=(1-$ $\left.\Psi_{t, 2}\right) / 2, \Psi_{p, 2}=0$, and $\Psi_{p, 1}=-\Psi_{p, 3}=-\mu_{2} \Psi_{t, 2} \tilde{x}_{1}$. The constraint on the poloidal fluxes $\Psi_{p, 2}$ and $\Psi_{p, 3}$ ensures that the magnetic field is continuous across the interfaces, thereby implying that the sole current sheet is within the inner volume. The amplitude of the current density is proportional to $\mu_{2}$ and is chosen to be small enough, $\mu_{2} \tilde{x}_{2} \ll 1$, such that we are in the strong guide field limit. We remark that the exact value of $\mu_{2}$ is irrelevant since the tearing mode stability is expected to be independent of the amplitude of the current, i.e., $\Delta^{\prime}$ is independent of $j_{z 0}$, see Eq. (8). Finally, the toroidal flux enclosed by the current sheet, $\Psi_{t, 2}$, can be varied in order to modify the equilibrium aspect ratio since we expect that $\delta / L \sim \Psi_{t, 2}$.

The stability of these MRxMHD equilibria can be assessed by studying the derivative of the interface force balance, $f_{l}$, with respect to poloidal perturbations in the interface geometry, $x_{l}$. Writing the perturbed forces and geometry in Fourier series, $f_{l}=\sum_{m} f_{l, m} \cos (m \theta)$ and $x_{l}=\sum_{m} x_{l, m} \cos (m \theta)$, where $\theta=2 \pi y / L$, we are interested in the matrix elements

$$
H_{i j}=\frac{\partial f_{l_{i}, m_{i}}}{\partial x_{l_{j}, m_{j}}}
$$

where $l_{q}=\lfloor 1+(q-1) / \mathcal{M}\rfloor$ and $m_{q}=q-1-\left(l_{q}-1\right) \mathcal{M}$, for $q=1 \ldots(N-1) \mathcal{M}$, with $N$ the number of relaxed volumes and $\mathcal{M}$ the number of Fourier modes. When evaluated at fixed magnetic helicity and fluxes, the Hessian matrix, $H$, represents the second variation of the MRxMHD energy functional. Hence the eigenvalues of $H$ provide information about the stability of each perturbation eigenmode.

The equilibrium magnetic helicity, Eq. (2), in each volume can be calculated from Eqs. (10) and (11) and is given by

$$
K_{l}=\left(\Psi_{t, l}^{2}+\Psi_{p, l}^{2}\right) \frac{\bar{\mu}_{l}}{2 \sin ^{2}\left(\bar{\mu}_{l}\right)}
$$

and thus the conservation of fluxes and helicity also implies the conservation of $\bar{\mu}_{l}$.

Considering first that only $m=0,1$ modes exist, an analytical expression for the Hessian can be obtained by expressing $f_{l, m}$ in terms of $x_{l^{\prime}, m^{\prime}}$ at fixed magnetic helicity and fluxes. The $m=0$ component of the forces, $f_{l, 0}$, was already obtained in Eq. (12) and we only need to express it by using the constraint of conserved helicity. That is,

$$
f_{l, 0}=\frac{1}{4 L^{2}}\left[\frac{\bar{\mu}_{l+1} K_{l+1}}{\tilde{x}_{l+1,0}^{2}}-\frac{\bar{\mu}_{l} K_{l}}{\tilde{x}_{l, 0}^{2}}\right] .
$$

and we immediately see that $\partial f_{l, 0} / \partial x_{l, 1}=0$. The $m=1$ component of the forces was calculated in [10] and is given by

$$
f_{l, 1}=\left(\mathcal{C}_{l+1}^{-}-\mathcal{C}_{l}^{+}\right) x_{l, 1}-\mathcal{D}_{l+1} x_{l+1,1}-\mathcal{D}_{l} x_{l-1,1}
$$

where $\mathcal{C}_{l}^{ \pm}$and $\mathcal{D}_{l}$ are complicated functions of the equilibrium whose exact expression can be found in [10]. Since at equilbrium $x_{l, 1}=0$, we have that $\partial f_{l, 1} / \partial x_{l, 0}=0$. Therefore the Hessian is

$$
\begin{aligned}
H & =\left[\begin{array}{cccc}
\frac{\partial f_{1,0}}{\partial x_{1,0}} & 0 & \frac{\partial f_{1,0}}{\partial x_{2,0}} & 0 \\
0 & \frac{\partial f_{1,1}}{\partial x_{1,1}} & 0 & \frac{\partial f_{1,1}}{\partial x_{2,1}} \\
\frac{\partial f_{2,0}}{\partial x_{1,0}} & 0 & \frac{\partial f_{2,0}}{\partial x_{2,0}} & 0 \\
0 & \frac{\partial f_{2,1}}{\partial x_{1,1}} & 0 & \frac{\partial f_{2,1}}{\partial x_{2,1}}
\end{array}\right] \\
& =\left[\begin{array}{cccc}
\mathcal{A}_{1}+\mathcal{A}_{2} & 0 & -\mathcal{A}_{2} & 0 \\
0 & \mathcal{C}_{2}^{-}-\mathcal{C}_{1}^{+} & 0 & -\mathcal{D}_{2} \\
-\mathcal{A}_{2} & 0 & \mathcal{A}_{2}+\mathcal{A}_{3} & 0 \\
0 & -\mathcal{D}_{2} & 0 & \mathcal{C}_{3}^{-}-\mathcal{C}_{2}^{+}
\end{array}\right]
\end{aligned}
$$

where $\mathcal{A}_{l}=\bar{\mu}_{l} K_{l} /\left(4 L^{2} \tilde{x}_{l, 0}^{3}\right)$.

When evaluating numerically the eigenvalues of the Hessian, Eq. (19), we discover that they are all positive except one, $\lambda$, which becomes negative for sufficiently small current sheet aspect ratio. The corresponding eigenvector is $\left(x_{1,0}, x_{1,1}, x_{2,0}, x_{2,1}\right)_{\lambda}=(0,1,0,-1)$, namely, a pure $m=1$ perturbation of the two internal interfaces with an amplitude equal and opposite. Figure 2 shows the dependence of $\lambda$ on $\delta / L$. The value of $\delta / L$ at which $\lambda$ changes sign coincides with that at which $\Delta^{\prime}$ changes sign. Since $\lambda<0$ implies that the mode is unstable, we have just shown that the MRxMHD stability boundary is in exact agreement with linear tearing mode theory.

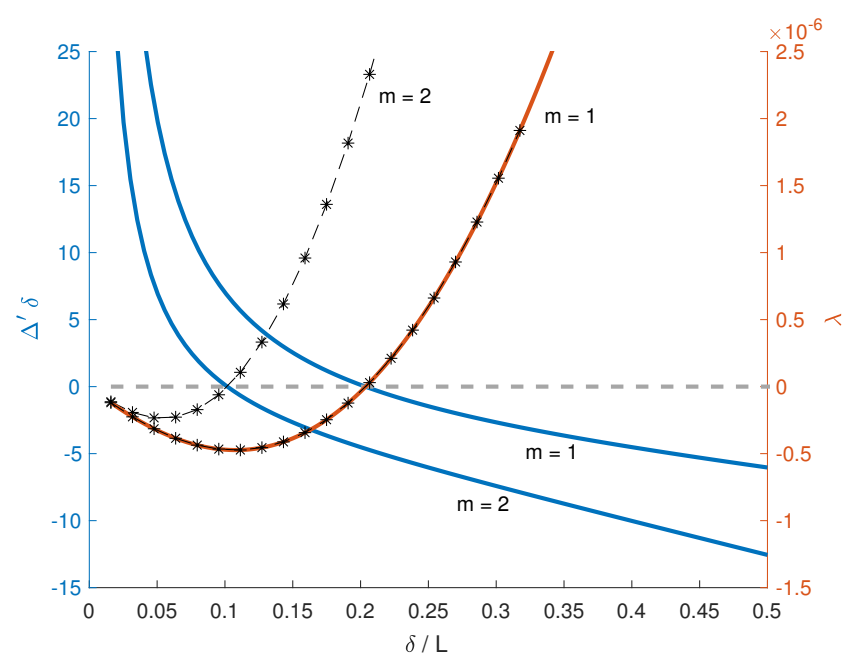

FIG. 2: Solid blue curves: normalized threshold parameter for the tearing mode instability, $\Delta^{\prime} \delta$, as a function of the current sheet aspect ratio, $\delta / L$, for the $m=1$ and $m=2$ modes. Curves obtained from Eq. (8). Solid orange curve: smallest eigenvalue, $\lambda$, of the MRxMHD Hessian as a function of the current sheet aspect ratio, $\delta / L$, as calculated from Eq. (19). Dashed lines with stars: eigenvalues of the MRxMHD Hessian as obtained from SPEC for the $m=1$ and $m=2$ modes.

We can also show analytically that the condition for the $m=1$ instability, $\lambda<0$, corresponds to the tearing mode onset condition, Eq. (9). In fact, we have that

$$
\lambda=\mathcal{D}_{2}+\mathcal{C}_{2}^{-}-\mathcal{C}_{1}^{+} .
$$


In the limit of a strong guide field, $\bar{\mu}_{2} \ll 1$, and using the fact that $\mu_{1}=\mu_{3}=0, \Psi_{p, 2}=0$, and $\Psi_{p, 1}=-\Psi_{p, 3}=$ $-\mu_{2} \Psi_{t, 2} \tilde{x}_{1}$, we have that

$$
\begin{aligned}
\mathcal{C}_{1}^{ \pm} & =\mathcal{C}_{3}^{ \pm}=\mp \frac{\mu_{2}^{2} \Psi_{t, 2}^{2}}{L^{3}} \frac{\pi}{2} \operatorname{coth}\left(4 \pi \tilde{x}_{1,0} / L\right) \\
\mathcal{C}_{2}^{ \pm} & =\frac{\mu_{2}^{2} \Psi_{t, 2}^{2}}{4 L^{2}} \frac{1}{\tilde{x}_{2,0}}\left( \pm 1 \mp \sigma \tilde{x}_{2,0} \operatorname{coth}\left(2 \sigma \tilde{x}_{2,0}\right)\right) \\
\mathcal{D}_{2} & =-\frac{\mu_{2}^{2} \Psi_{t, 2}^{2}}{4 L^{2}} \frac{\sigma}{\sinh \left(2 \sigma \tilde{x}_{2,0}\right)}
\end{aligned}
$$

with $\sigma=(2 \pi / L) \sqrt{\left|\mu_{2}^{2} L^{2} / 4 \pi^{2}-1\right|} \approx 2 \pi / L$. Recognizing that $\tilde{x}_{2,0}=\delta / 2$ and considering a sufficiently large system, $\tilde{x}_{1,0} / L \gg 1$, the condition $\lambda<0$ becomes

$$
\frac{\delta}{L}\left(\cosh \left(2 \pi \frac{\delta}{L}\right)-1\right)+\left(\frac{\delta}{L}-\frac{1}{\pi}\right) \sinh \left(2 \pi \frac{\delta}{L}\right)<0
$$

which, to first order in $\delta / L$, gives $\delta / L<1 / \pi \approx 0.3$. The more accurate, numerical solution to Eq. (24) is $\delta / L \lesssim 0.203$ which is in agreement with the tearing mode prediciton, Eq. (9).

Finally, we can also use the SPEC code [17] to calculate the same MRxMHD equilibrium and the corresponding Hessian but with an arbitrary number of poloidal mode numbers. Figure 2 shows the eigenvalues obtained for the $m=1$ and $m=2$ modes, as a function of the aspect ratio $\delta / L$. The analytical result is recovered for the $m=1$ mode and the $m=2$ mode becomes unstable exactly as expected from tearing mode theory, namely at $\delta / L \lesssim 0.1$.

The fundamental result presented in this letter is that MRxMHD can be used to predict linear resistive stability of force-free equilibria. That is, resistive MHD stability can be obtained from a variational principle! We would like to notice that, even though we have considered here a particular current profile (with the advantage of being analytically tractable), we expect that the equivalence between tearing and MRxMHD stability persists for any current profile (described by piece-wise constant currents in MRxMHD), although this has to be tested numerically. However, the effect of pressure on stability still needs to be carefully investigated. In fact, it is very possible that the effect of pressure on tearing mode stability will be quite restrictive in $\mathrm{MRxMHD}$, given that the variational principle from which it derives contains, as in ideal MHD, some simplified equation of state relating pressure and density variations [17]. We therefore must address this question in future investigations.

An important implication of these findings is that numerical codes like SPEC, which calculate MRxMHD equilibria in toroidal geometry and can compute the Hessian with an arbitrary large number of modes, may be used to predict the resistive stability of tokamaks as well as that of arbitrarily shaped stellarators. Moreover, if an equilibrium is found unstable, the nonlinear saturation of the mode could be easily obtained by finding the nearby, lower energy state. In particular, this would provide a fast way of predicting saturated island widths. Future investigations will address this question.

We acknowledge very helpful discussions with Amitava Bhattacharjee, Per Helander, Alessandro Zocco and Jonathan Graves. This work has been carried out within the framework of the EUROfusion Consortium and has received funding from the Euratom research and training programme 2014 - 2018 and 2019 - 2020 under grant agreement No 633053. The views and opinions expressed herein do not necessarily reflect those of the European Commission. This work was also supported by a grant from the Simons Foundation/SFARI (560651, AB).
[1] M. J. Hole, S. R. Hudson, and R. L. Dewar. Stepped pressure profile equilibria in cylindrical plasmas via partial Taylor relaxation. Journal of Plasma Physics, $72(06): 1167,2006$.

[2] M.J Hole, S.R Hudson, and R.L Dewar. Equilibria and stability in partially relaxed plasmavacuum systems. $\mathrm{Nu}$ clear Fusion, 47(8):746-753, 2007.

[3] J. B. Taylor. Relaxation of toroidal plasma and generation of reverse magnetic fields. Physical Review Letters, 33(19):1139-1141, 1974.

[4] J P Freidberg. ideal MHD. Cambridge University Press, 2014.

[5] M. D. Kruskal and R. M. Kulsrud. Equilibrium of a Magnetically Confined Plasma in a Toroid. Physics of Fluids, 1(4):265, 1958.

[6] G. R. Dennis, S. R. Hudson, R. L. Dewar, and M. J. Hole. The infinite interface limit of multiple-region relaxed magnetohydrodynamics. Physics of Plasmas, 20(3), 2013
[7] J Loizu, S R Hudson, C Nührenberg, J Geiger, and P Helander. Equilibrium $\beta$-limits in classical stellarators. Journal of Plasma Physics, 83, 2017.

[8] Oscar P. Bruno and Peter Laurence. Existence of threedimensional toroidal MHD equilibria with nonconstant pressure. Communications on Pure and Applied Mathematics, 49(7):717-764, 1996.

[9] J. Loizu, S. R. Hudson, A. Bhattacharjee, S. Lazerson, and P. Helander. Existence of three-dimensional idealmagnetohydrodynamic equilibria with current sheets. Physics of Plasmas, 22(9):090704, sep 2015.

[10] J. Loizu, S. Hudson, A. Bhattacharjee, and P. Helander. Magnetic islands and singular currents at rational surfaces in three-dimensional magnetohydrodynamic equilibria. Physics of Plasmas, 22(2):022501, feb 2015.

[11] B F Kraus and S.R Hudson. Theory and discretization of ideal magnetohydrodynamic equilibria with fractal pressure profiles. Physics of Plasmas, 24, 2017.

[12] S R Hudson and B F Kraus. Three-dimensional mag- 
netohydrodynamic equilibria with continuous magnetic fields. Journal of Plasma Physics, 83, 2017.

[13] J. Loizu, S. R. Hudson, P. Helander, S. A. Lazerson, and A. Bhattacharjee. Pressure-driven amplification and penetration of resonant magnetic perturbations. Physics of Plasmas, 23(5), 2016.

[14] J. P. Goedbloed and R.Y. Dagazian. Kinks and tearing modes in simple configurations. Physical Review A, 4(4):1554-1560, 1971.
[15] R J Goldston and $\mathrm{P} \mathrm{H}$ Rutherford. Introduction to Plasma Physics. Institute of Physics Publishing, 1995.

[16] T. S. Hahm and R. M. Kulsrud. Forced magnetic reconnection. Physics of Fluids, 28(8):2412, 1985.

[17] S. R. Hudson, R. L. Dewar, G. Dennis, M. J. Hole, M. McGann, G. Von Nessi, and S. Lazerson. Computation of multi-region relaxed magnetohydrodynamic equilibria. Physics of Plasmas, 19(11), 2012. 